The Journal of Bone E Joint Surgery
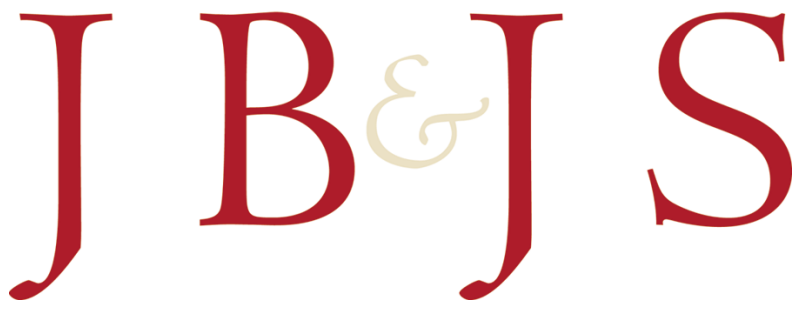

This is an enhanced PDF from The Journal of Bone and Joint Surgery

The PDF of the article you requested follows this cover page.

\title{
The Natural History of Pediatric Trigger Thumb
}

Goo Hyun Baek, Ji Hyeung Kim, Moon Sang Chung, Seung Baik Kang, Young Ho Lee and Hyun Sik Gong J Bone Joint Surg Am. 2008;90:980-985. doi:10.2106/JBJS.G.00296

This information is current as of December 23, 2009

Reprints and Permissions

Publisher Information
Click here to order reprints or request permission to use material from this article, or locate the article citation on jbjs.org and click on the [Reprints and Permissions] link.

The Journal of Bone and Joint Surgery

20 Pickering Street, Needham, MA 02492-3157

www.jbjs.org 


\title{
The Natural History of Pediatric Trigger Thumb
}

\author{
By Goo Hyun Baek, MD, Ji Hyeung Kim, MD, Moon Sang Chung, MD, Seung Baik Kang, MD, \\ Young Ho Lee, MD, and Hyun Sik Gong, MD
}

Investigation performed at the Department of Orthopedic Surgery, Seoul National University College of Medicine, Seoul, South Korea

\begin{abstract}
Background: Pediatric trigger thumb is a condition of flexion deformity of the interphalangeal joint in children. Although the surgical outcome is satisfactory, the indications for nonoperative treatment for this condition are not clear. The aim of the present study was to determine the rate of resolution of untreated pediatric trigger thumb.
\end{abstract}

\begin{abstract}
Methods: Data on seventy-one thumbs in fifty-three children were collected prospectively. The dates of the first visits ranged from April 1994 to March 2004. Patients were diagnosed with pediatric trigger thumb during initial outpatient department visits. During the present study, no treatment such as passive stretching or splinting was applied. The amount of flexion deformity at the thumb interphalangeal joint was measured at every six-month follow-up visit, and the duration of follow-up was at least two years after diagnosis. The end point of follow-up was when the deformity caused pain or secondary deformity or prevented normal use of the hand. The median duration of follow-up was forty-eight months.
\end{abstract}

Results: Of the seventy-one trigger thumbs, forty-five (63\%) resolved spontaneously. The median time from the initial visit to resolution was forty-eight months. There was no significant difference in the pattern of resolution between patients with unilateral and bilateral trigger thumb. Although resolution was not observed in the remaining twenty-six thumbs, flexion deformities improved in twenty-two thumbs. For the first two years after the initial visit, the mean flexion deformity significantly decreased over the one-year intervals $(p<0.05)$.

Conclusions: Pediatric trigger thumb can be expected to resolve without treatment in $>60 \%$ of patients. Moreover, the flexion deformity can be expected to show an improving pattern in patients who do not have resolution. This information may help both parents and surgeons to make decisions regarding the treatment of pediatric trigger thumb.

Level of Evidence: Prognostic Level IV. See Instructions to Authors for a complete description of levels of evidence.

$\mathrm{P}$ ediatric trigger thumb is a stenosing tenosynovitis of the flexor pollicis longus tendon with flexion deformity of the interphalangeal joint. Estimates of the prevalence have ranged from one per 2000 office visits $^{1}$ to $2.2 \%$ of all pediatric upper extremity anomalies ${ }^{2}$. The etiology of the condition referred to as pediatric trigger thumb is unclear, and controversy persists as to whether it is a congenital condition or an acquired condition. A congenital or hereditary cause has been proposed by several authors ${ }^{3,4}$; however, recent studies ${ }^{5,6}$ have supported the notion that it is acquired. Trauma has been proposed as a possible cause because pediatric trigger thumb can be misdiagnosed as a fracture of the thumb or as a dislocation of the interphalangeal joint ${ }^{7}$. Characteristically, a nodule is palpable on the tendon in the region of the metacarpal head in association with thickening of the fibrous tendon sheath. In addition, a flexion deformity of the interphalangeal joint is observed in most cases.

Definitive treatment of this condition usually has been operative $^{1,4,8,9}$. However, Dinham and Meggitt observed that patients presenting at birth had a spontaneous recovery rate of $30 \%$ and that those presenting between six and thirty months of age had a spontaneous recovery rate of $12 \%{ }^{3}$. Several authors have also suggested a nonoperative approach for the treatment of pediatric trigger thumb $b^{10-17}$.

In the present study, patients with a diagnosis of pediatric trigger thumb were prospectively observed for at least two years, over a period ranging from twenty-four to 114 months, and the degree of flexion deformity of the interphalangeal joint was measured periodically. The objective of the present study was to establish the natural history of pediatric trigger thumb.

Disclosure: The authors did not receive any outside funding or grants in support of their research for or preparation of this work. Neither they nor a member of their immediate families received payments or other benefits or a commitment or agreement to provide such benefits from a commercial entity. No commercial entity paid or directed, or agreed to pay or direct, any benefits to any research fund, foundation, division, center, clinical practice, or other charitable or nonprofit organization with which the authors, or a member of their immediate families, are affiliated or associated. 


\section{Materials and Methods}

$\mathrm{T}$ his prospective study included all of the patients with pediatric trigger thumb who visited the pediatric hand service at our Children's Hospital between April 1994 and March 2004. The inclusion criteria were (1) a diagnosis of pediatric trigger thumb on the basis of the history and a physical examination, (2) no operative or nonoperative treatment, and (3) normal findings on radiographs of the thumb. Children who underwent operative treatment during the observation period, for any reason, were excluded. We obtained institutional review board approval for the present study. We adequately explained to the parents about the condition, the outcome of operative treatment, and possible problems related to the delay of operative treatment, and we obtained informed consent before the patients were enrolled in the study. We specified the end point of observation as when the deformity caused pain or secondary deformity or prevented the normal use of the hand.

Flexion of the interphalangeal joint was measured every six months by a single physician (G.H.B.) with use of a goniometer, with the wrist held in neutral extension, the thumb in $20^{\circ}$ of palmar abduction, and the metacarpophalangeal joint in neutral extension. Changes in the amount of interphalangeal joint flexion were analyzed over time. To determine the significance of these changes, we used repeated measures analysis of variance followed by Bonferroni analyses. As there are no standard definitions of resolution of trigger thumb in the literature, we defined resolution as having occurred when the interphalangeal joint flexion deformity became $0^{\circ}$. Even if the normal side had hyperextension of this joint, we thought that the affected thumb did not need to have the same hyperextension as the normal side from a functional or aesthetic aspect.

The rates of resolution were determined, and the average time from the initial visit to resolution was estimated with use of a Kaplan-Meier survival analysis, with resolution of trigger thumb as the end point. We also stratified our patients into groups according to whether they had unilateral or bilateral involvement, and survival analysis was performed for each group. To determine any significant difference between these survival curves, we used the log-rank test. The level of significance was set at $\mathrm{p}<0.05$.

\section{Results}

Ceventy-three thumbs in fifty-five patients (thirty boys and $\checkmark$ twenty-five girls) were initially enrolled. Two of these fiftyfive children were managed with A1 pulley releases. One of these two patients was a twenty-five-month-old girl, and the flexion deformity of the left thumb had not changed after sixteen months. The other patient was a seventeen-month-old boy who had a left congenital trigger thumb with a $45^{\circ}$ flexion deformity, which had improved to $30^{\circ}$ five years later. The parents of these two patients requested operative treatment, which was performed at the ages of forty-one months and seventy-four months, respectively. Postoperatively, neither child had a flexion deformity at the interphalangeal joint, and these two children were excluded in accord with the study criteria.

\begin{tabular}{|c|c|c|}
\hline Time of Follow-up (yr) & $\begin{array}{l}\text { No. of } \\
\text { Thumbs }\end{array}$ & $\begin{array}{l}\text { Mean Flexion } \\
\text { Deformity (deg) }\end{array}$ \\
\hline Initial visit & 71 & 26.5 \\
\hline 0.5 & 71 & 23.0 \\
\hline 1.0 & 71 & 18.7 \\
\hline 1.5 & 71 & 15.3 \\
\hline 2.0 & 71 & 12.4 \\
\hline 2.5 & 57 & 11.8 \\
\hline 3.0 & 49 & 11.1 \\
\hline 3.5 & 47 & 10.3 \\
\hline 4.0 & 41 & 6.9 \\
\hline 4.5 & 28 & 6.0 \\
\hline 5.0 & 20 & 6.7 \\
\hline 5.5 & 19 & 7.1 \\
\hline 6.0 & 16 & 5.9 \\
\hline 6.5 & 12 & 5.8 \\
\hline 7.0 & 7 & 4.0 \\
\hline 7.5 & 6 & 2.7 \\
\hline 8.0 & 6 & 2.7 \\
\hline 8.5 & 6 & 2.7 \\
\hline 9.0 & 6 & 0.7 \\
\hline 9.5 & 1 & 0 \\
\hline
\end{tabular}

Thus, fifty-three children (seventy-one thumbs) constituted the study cohort. The median age of the patients at the time of the first visit was twenty-three months (range, one to fifty-five months; standard deviation, 11.9 months), and the median duration of follow-up was forty-eight months (range, twenty-four to 114 months; standard deviation, 24.4 months). The seventy-one thumbs included twenty-three thumbs in twenty-three patients with unilateral involvement, thirty-six thumbs in eighteen patients with bilateral involvement, and twelve thumbs in twelve patients who presented with unilateral trigger thumb with a history of resolution on the other side.

The average initial flexion deformity of the interphalangeal joint for the seventy-one thumbs was $26.2^{\circ}$ (range, $15^{\circ}$ to $\left.45^{\circ}\right)$. Overall, trigger thumb resolved in forty-five $(63 \%)$ of the seventy-one thumbs. The number of thumbs at each followup period and the mean flexion deformity of the interphalangeal joint are presented in Table I. Twenty-eight thumbs were followed for more than four years, and forty-three thumbs were followed for two to four years.

In sixty-seven thumbs (94\%), the flexion deformity decreased. The average amount of flexion deformity decreased over time, except during the one-year period from 4.5 to 5.5 years after the time of presentation (Fig. 1). Repeated-measures analysis of variance showed that there were significant differences over time among the mean flexion deformities of the interphalangeal joint at every six-month follow-up visit 


\section{Change in the average amount of IP joint flexion deformity over time}

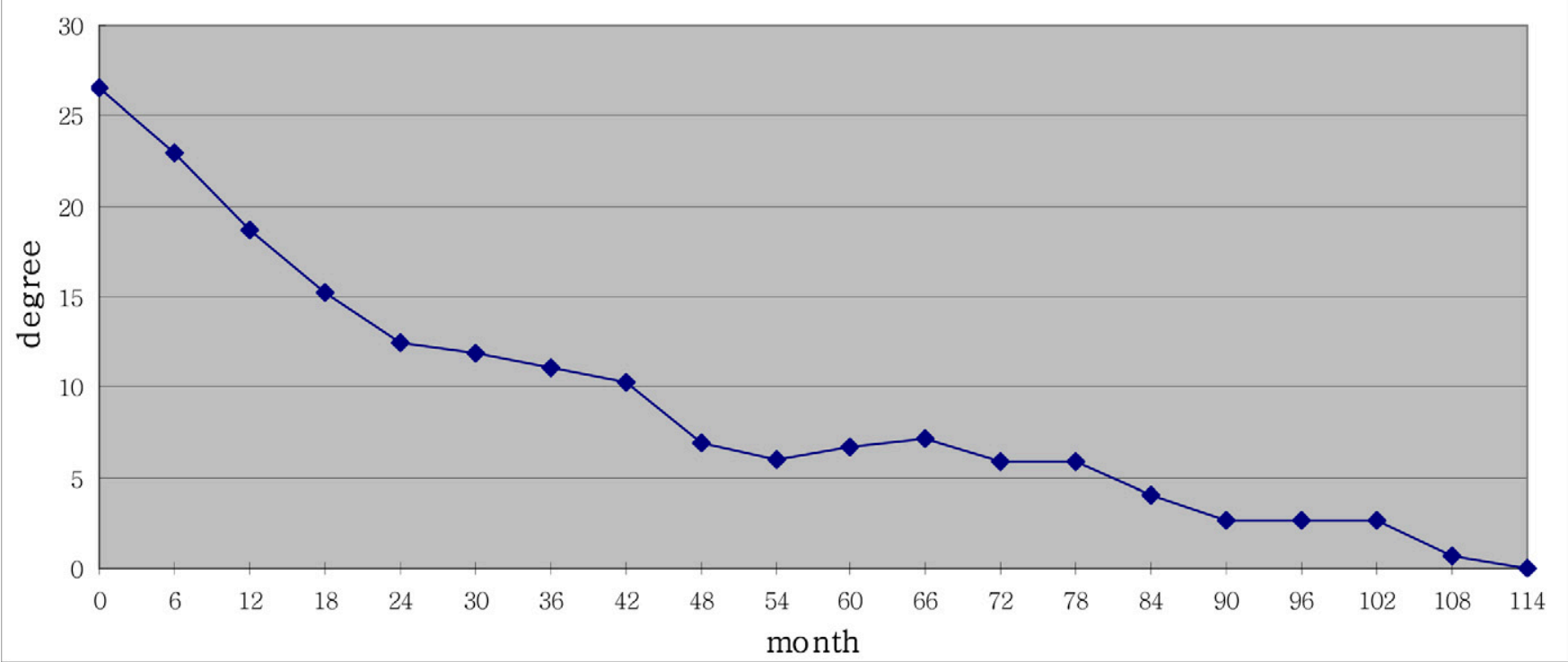

Fig. 1

Line graph illustrating the changes in the average amount of flexion deformity at six-month follow-up intervals. The average amount of flexion deformity showed a decreasing pattern except between 4.5 and 5.5 years after the time of the initial examination. For the first two years after the initial visit, the average amount of flexion deformity decreased significantly every one-year interval. Subsequently, no significant difference was found between oneyear intervals, although a steadily decreasing trend was apparent. IP = interphalangeal.

$(\mathrm{p}<0.001)$. Post hoc Bonferroni tests indicated that for the first two years after the initial visit, the average flexion deformity decreased significantly every one-year interval $(\mathrm{p}<0.05)$, but no significant changes were found thereafter.

In the group of eighteen children who presented with bilateral involvement, ten had resolution of the flexion deformity on both sides, five had resolution on one side, and three had persistence of bilateral deformity. In the group of twelve children who presented with a flexion deformity on one side with a history of resolution on the other, four had resolution of the flexion deformity. In the group of fifteen children who presented with unilateral deformity of the right thumb only, ten had resolution, and in the group of eight children who presented with unilateral deformity of the left thumb only, six had resolution.

Resolution did not occur in twenty-six thumbs. However, we observed that twenty-two $(85 \%)$ of these twenty-six thumbs had an improvement in the flexion deformity as measured by a change of at least $5^{\circ}$. In this group, the initial flexion deformity averaged $27.7^{\circ}$ (range, $15^{\circ}$ to $45^{\circ}$ ) and the final flexion deformity averaged $15.6^{\circ}$ (range, $5^{\circ}$ to $40^{\circ}$ ). The paired $t$ test indicated that the change in flexion deformity between the initial and final visits in the group without resolution was significant $(p<0.01)$. At the time of the present report, none of these twenty-six thumbs had been operatively treated. No parent had requested operative treatment because most of the children had improvement, and the parents of some children without improvement wanted to wait longer.

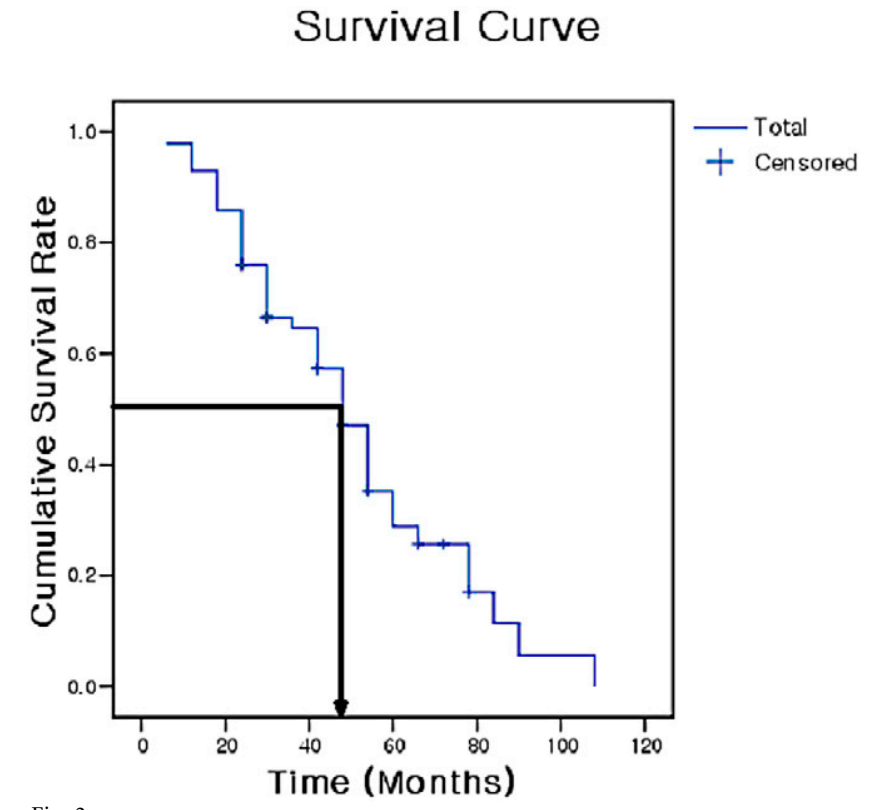

Fig. 2

Kaplan-Meier survival curve, with resolution of trigger thumb as the end point. The arrow indicates that the median survival time (the median time of having trigger thumb) from the initial visit to the time of resolution was forty-eight months (95\% confidence interval, 41.0 to 55.1 months). This means that half of the cohort had resolution by about four years after the initial visit. 


\section{Survival Curve}

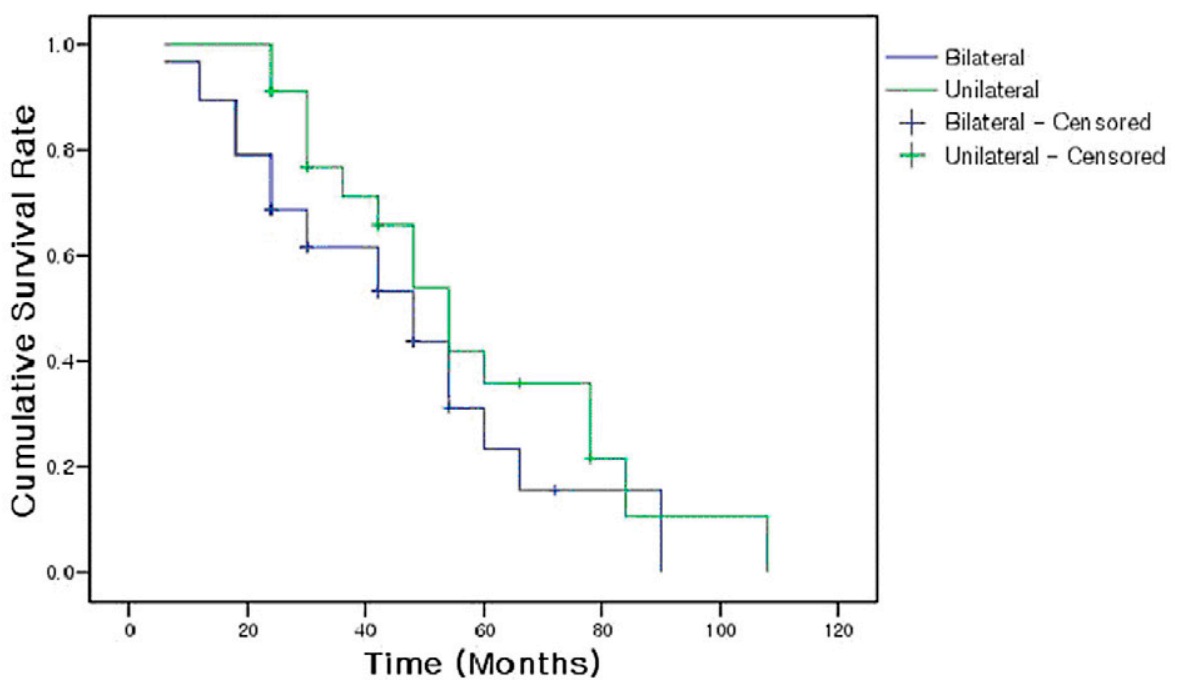

Fig. 3

Kaplan-Meier survival curves for the groups with unilateral and bilateral involvement. In the unilateral group, the median time from the initial visit to resolution was fifty-four months (95\% confidence interval, forty-three to sixty-five months). In the bilateral group, the median time from the initial visit to resolution was forty-eight months ( $95 \%$ confidence interval, thirty-two to sixty-four months). When comparing these survival curves with use of the log-rank test, a significant difference was not found.

None of these children had development of serious compensatory metacarpophalangeal hyperextension affecting daily use of the thumb.

In the group of forty-five thumbs that had resolution, the average age at the time of resolution was 60.1 months (range, twenty-four to 128 months). Kaplan-Meier analysis revealed that the overall median time from the initial visit to resolution was forty-eight months $(95 \%$ confidence interval, 41.0 to 55.1 months) (Fig. 2), meaning that half of the cohort had resolution by four years. In the group with unilateral involvement, the median time from the initial visit to resolution was fifty-four months ( $95 \%$ confidence interval, forty-three to sixty-five months). In the group with bilateral involvement, the median time from the initial visit to resolution was forty-eight months $(95 \%$ confidence interval, thirty-two to sixty-four months). The log-rank test indicated that there was not a significant difference between these two survival curves $(p>0.05)$ (Fig. 3).

\section{Discussion}

$\mathrm{T}$ he treatment of pediatric trigger thumb remains controversial, although over the years, treatment modalities have changed from operative treatment only to nonoperative combined with operative treatment. In 1949, Sprecher reported on a series of pediatric patients with trigger thumb ${ }^{18}$. Some patients underwent an initial period of nonoperative treatment, but the results were unsatisfactory. In particular, it was found that results of splint therapy were disappointing. Eventually, operative treatment was performed in all cases. In 1954, Fahey and Bollinger evaluated twelve children with pediatric trigger thumb and concluded that only operative treatment produced satisfactory results. ${ }^{8}$. They emphasized that local excision of the tendon sheath and encouragement of early motion were important. In 1966, Patel also supported the operative treatment of pediatric trigger thumb in a series of ten pediatric cases ${ }^{19}$. In 1974, Dinham and Meggitt established treatment guidelines for trigger deformity, specifically, that (1) a trigger deformity that is present at birth should be carefully observed for twelve months, (2) a trigger deformity that is first noticed between the ages of six and thirty months should be observed for six months, and (3) operative treatment should be performed before the age of four years to prevent the creation of residual flexion deformities ${ }^{3}$. However, Skov et al. later concluded that the risk of residual flexion deformity was no higher following operations conducted in patients who were more than three years old, supporting initial nonoperative treatment regardless of age ${ }^{20}$.

Several conservative approaches to the treatment of pediatric trigger thumb with use of splints have been described by Japanese groups ${ }^{10-13}$, with success rates ranging from $48 \%$ to 89\%. In 1998, Mulpruek and Prichasuk evaluated forty-two children to determine the rate of spontaneous recovery and treatment outcome ${ }^{21}$. In that study, ten patients $(24 \%)$ had spontaneous recovery within three months after the initial visit and thirty-two patients underwent operative release. Delaying surgery beyond the age of three years did not seem to affect the 
outcome. In 2001, Watanabe et al. treated sixty trigger thumbs with passive stretching exercises and reported satisfactory results in $96 \%$ of the cases ${ }^{14}$.

Many authors have addressed the natural history of pediatric trigger thumb. In the study by Dinham and Meggitt, the rate of resolution of pediatric trigger thumb was $31 \%$ when the deformity was noted at birth and $12 \%$ when the deformity was first noted between the ages of six and thirty months ${ }^{3}$. The lengths of the observation periods in these two groups were twelve and six months, respectively. Ger et al., in a study of forty-one children (fifty-three thumbs), administered forty months of nonoperative therapy to those who were diagnosed before the age of six months but found no case of resolution'. Similarly, Steenwerckx et al. evaluated forty-one patients (fiftyseven digits) and noted that none of the patients with trigger thumb had spontaneous recovery over six months ${ }^{22}$. Conversely, Dunsmuir and Sherlock reported a $49 \%$ rate of resolution over a mean duration of follow-up of seven months and concluded that a more conservative approach to treatment might prevent unnecessary operations ${ }^{15}$. Moon et al. reported that twelve $(34 \%)$ of thirty-five cases of congenital trigger thumb resolved spontaneously after a mean duration of conservative treatment of five months ${ }^{16}$, and Tan et al. reported an overall success rate of $66 \%$ after a mean duration of nonoperative treatment of 5.9 months ${ }^{17}$. However, the above studies were retrospective in nature and had short durations of follow-up, which may explain the lower rates of resolution that were observed as compared with the results from the present study, in which the mean duration of follow-up was forty-nine months.

The present study was planned on the basis of a clinical impression that we had had, prior to the study, that many cases of pediatric trigger thumb resolved spontaneously. Furthermore, it has been our experience that flexion deformities that are treated operatively respond regardless of the age of the patient at the time of the operation. Thus, we were able to design the present prospective study with confidence and were able to observe the changes in the amounts of flexion deformity and the rates of resolution without treatment of any type.

On the basis of the present study, we cannot determine how long one can safely observe these patients. Skov et al. noted that the risk of residual deformity was no higher even if operative treatment was performed in patients over the age of three year $\mathrm{s}^{20}$. Herdem et al. reported that a radial deviation deformity had developed in two patients who were managed late (at ten and thirteen years of age) and noted that this deformity is difficult to detect in the flexed thumb but can be seen when the flexion deformity is corrected ${ }^{23}$. Moreover, fine motor development in early childhood may be adversely affected by these primary and secondary deformities, and these issues should be discussed with the parents before proceeding with nonoperative treatment. However, none of our patients had development of radial deviation at the interphalangeal joint or serious compensatory metacarpophalangeal hyperextension that hampered writing or daily use of the thumb.

The present study had several limitations. First, there was a risk of referral bias in our sample, as the patients generally were referred to our pediatric hand center and the study involved a hospital-based cohort. This method may have left out patients who were not noted to have a trigger thumb, patients who did not have access to medical care, or patients who refused to be seen. However, as more severe cases usually tend to be referred to our hand center, the present study may not overestimate the rate of resolution of pediatric trigger thumb in the general population. Second, because of the concerns of recall bias, we did not analyze the duration of trigger thumb prior to entry into the study. Therefore, the present study does not represent the real duration of the condition or the true natural history of trigger thumb. Third, we did not study intraobserver variability in the measurement of the flexion deformity of the thumb interphalangeal joint. One experienced treating physician measured the angle throughout the period of the study. This factor may have reduced the variability of the measurements but also may have created a bias in data collection. However, despite possible intraobserver variability and bias, the final rate of resolution should not have been affected because resolution is simple to determine. Last, the present study involved only Korean children and may not represent the general characteristics of pediatric trigger thumb in other populations. Although previous studies demonstrating high rates of resolution after nonoperative treatment were from both Eastern ${ }^{17}$ and Western ${ }^{15}$ countries, there may be an ethnic difference in the prevalence of pediatric trigger thumb among various populations.

Our results may help both parents and surgeons to make decisions regarding the treatment of pediatric trigger thumb. A high rate of resolution as well as the possibility that the duration of nonoperative care can be long should be discussed so that parents may take part in the decision regarding the use of nonoperative as opposed to operative treatment.

Goo Hyun Baek, MD

Ji Hyeung Kim, MD

Moon Sang Chung, MD

Seung Baik Kang, MD

Young Ho Lee, MD

Hyun Sik Gong, MD

Department of Orthopedic Surgery,

Seoul National University College of Medicine,

28 Yongon-dong, Chongno-gu, Seoul 110-744, South Korea.

E-mail address for G.H. Baek: ghbaek@snu.ac.kr

\section{References}

1. Ger E, Kupcha $P$, Ger D. The management of trigger thumb in children. J Hand Surg [Am]. 1991;16:944-7.
2. Flatt AE. The care of congenital hand anomalies. 2nd ed. St. Louis: Quality Medical Publishing; 1994. p 59-60. 
The Journal of Bone \& Joint Surgery $\cdot$ Jbjs.org VOlume 90 -A $\cdot$ Number $5 \cdot$ May 2008

3. Dinham JM, Meggitt BF. Trigger thumbs in children. A review of the natural history and indications for treatment in 105 patients. J Bone Joint Surg Br. 1974;56:153-5.

4. van Genechten F. Familial trigger thumb in children. Hand. 1982;14:56-8.

5. Rodgers WB, Waters PM. Incidence of trigger digits in newborns. J Hand Surg [Am]. 1994;19:364-8.

6. Slakey JB, Hennrikus WL. Acquired thumb flexion contracture in children: congenital trigger thumb. J Bone Joint Surg Br. 1996;78:481-3.

7. Weilby A. Trigger finger. Incidence in children and adults and the possibility of a predisposition in certain age groups. Acta Orthop Scand. 1970;41:419-27.

8. Fahey JJ, Bollinger JA. Trigger-finger in adults and children. J Bone Joint Surg Am. 1954;36:1200-18.

9. White JW, Jensen WE. Trigger thumb in infants. AMA Am J Dis Child. 1953;85:141-5.

10. Ishikura T, Murakami T, Kumagai S, Suzuki K, Suga Y. Conservative treatment of trigger thumbs in children. Journal of the Japanese Society for Surgery of the Hand. 1984;1:349-52 (in Japanese).

11. Negoro $\mathrm{H}$, Shioda $\mathrm{M}$, Hatanaka I, Nakano K. Conservative treatment of trigger thumbs. Seikeigeka. 1981;32:1732-4 (in Japanese).

12. Tsushima S, Ohmizo M. Splinting of trigger thumb. Sagyoryoho (Occupational therapy). 1989;8:610-6 (in Japanese).
The Natural History of Pediatric Trigger Thumb

13. Nemoto $K$, Nemoto $T$, Terada $N$, Amako $M$, Kawaguchi M. Splint therapy for trigger thumb and finger in children. J Hand Surg [Br]. 1996;21:416-8.

14. Watanabe $\mathrm{H}$, Hamada $\mathrm{Y}$, Toshima $\mathrm{T}$, Nagasawa $\mathrm{K}$. Conservative treatment for trigger thumb in children. Arch Orthop Trauma Surg. 2001;121:388-90.

15. Dunsmuir RA, Sherlock DA. The outcome of treatment of trigger thumb in children. J Bone Joint Surg Br. 2000;82:736-8.

16. Moon WN, Suh SW, Kim IC. Trigger digits in children. J Hand Surg [Br]. 2001;26:11-2.

17. Tan AH, Lam KS, Lee EH. The treatment outcome of trigger thumb in children. J Pediatr Orthop B. 2002;11:256-9.

18. Sprecher EE. Trigger thumb in infants. J Bone Joint Surg Am. 1949;31:672-4.

19. Patel AP. Trigger thumb in infancy. Postgrad Med J. 1966;42:512-3.

20. Skov $O$, Bach A, Hammer A. Trigger thumbs in children: a follow-up study of 37 children below 15 years of age. J Hand Surg [Br]. 1990;15:466-7.

21. Mulpruek $P$, Prichasuk $S$. Spontaneous recovery of trigger thumbs in children. J Hand Surg [Br]. 1998;23:255-7.

22. Steenwerckx A, De Smet L, Fabry G. Congenital trigger digit. J Hand Surg [Am]. 1996;21:909-11.

23. Herdem $M$, Bayram $H$, Toğrul $E$, Sarpel $Y$. Clinical analysis of the trigger thumb of childhood. Turk J Pediatr. 2003;45:237-9. 